\title{
El falso problema de la privatización educativa a la luz de la crisis orgánica: Argentina, 2003-2015*
}

\author{
Eduardo Sartelli* \\ Romina De Luca ${ }^{* * *}$
}

Recibido: 26 de febrero de 2018

Evaluado: 4 de abril de 2018

Aceptado: 23 de octubre de 2018

Citar como: Sartelli, E. y De Luca, R. (2019). El falso problema de la privatización educativa a la luz de la crisis orgánica: Argentina, 20032015. Hallazgos, 16(31), 91-117. Doi: https://doi.org/10.15332/s1794-38 41.2019.0031.04

\section{Resumen}

Este artículo analiza la evolución de la matrícula pública y privada del nivel primario y secundario en Argentina en el periodo de 2003 a 2014. Se discute con otros trabajos académicos que sostienen que dicho periodo coincide con un aumento en los niveles de privatización educativa. Se muestra, a partir del cruce de datos educativos, de rendimiento educacional y poblacionales, que la evolución de la matrícula privada no acelera su ritmo y su expansión sigue el ciclo económico. Por su parte, se enseña cómo la matrícula estatal se ve condicionada por la evolución de los indicadores de rendimiento interno y la política educativa. En el nivel secundario, se concluye que la tendencia se corresponde con una mayor estatización y fragmentación del nivel. Se sostiene a modo de hipótesis que los subregistros poblacionales y las mejoras en el rendimiento interno escolar impiden ver la mayor estatización del sistema educativo argentino y los cambios que operan en las distintas modalidades educativas. Se sostiene que estos cambios guardan relación con las transformaciones y la crisis orgánica de la sociedad en un plano general. Desde una perspectiva teórica materialista, se entiende la dinámica escolar atravesada por los cambios en las relaciones sociales de producción, situación que explica la creciente degradación y estatización escolar.

Palabras clave: sistema educativo, privatización, tendencias, estatización.

\footnotetext{
Artículo de investigación resultado del proyecto UBACyT: "Expansión y profundización del régimen de gran industria en la Argentina y la conformación de una sobrepoblación relativa (1976 hasta la actualidad)". Director: Eduardo Sartelli. Duración: 2014-2017. Código: 20020100100927.

* Universidad de Buenos Aires, Argentina. Correo electrónico: eduardo.sartelli@yahoo.com.ar ORCID: https://orcid.org/0000-0003-2147-3424

*** Universidad de Buenos Aires, Argentina. Correo electrónico: rom.deluca@gmail.com ORCID: https://orcid.org/0000-0003-3805-6512
} 


\section{The false problem of educational privatization in light of the organic crisis: Argentina, 2003-2015}

\begin{abstract}
This article analyzes the evolution of public and private enrollment at the primary and secondary level in Argentina in the period from 2003 to 2014. It discusses with other academic studies that assert that this period coincides with an increase in the levels of educational privatization. It shows, from crisscrossing educational data, educational and population performance, that the evolution of private enrollment does not accelerate its pace and its expansion follows the economic cycle. On the other hand, it is shown how the state enrollment is conditioned by the evolution of internal performance indicators and educational policy. At the secondary level, it is concluded that the trend corresponds to a greater nationalization and fragmentation of the level. It is affirmed as a hypothesis that the population sub-registries and improvements in the internal school performance prevent seeing the greater nationalization of the Argentine educational system and the changes that operate in the different educational modalities. It is argued that these changes are related to the transformations and the organic crisis of society at a general level. From a materialist theoretical perspective, it is understood the school dynamics traversed by the changes in the social relations of production, situation that explains the growing degradation and school nationalization.
\end{abstract}

Keywords: educational system, privatization, trends, nationalization.
Received: February 26, 2018

Evaluated: April 4, 2018

Accepted: October 23, 2018 


\section{O falso problema da privatização educativa à luz da crise orgânica: Argentina 2003 - 2015}

Recebido: 26 de fevereiro de 2018

Avaliado: 4 de abril de 2018

Aceito: 23 de outubro de 2018

\section{Resumo}

Este artigo analisa a evolução da matrícula publica e privada do nível primário e secundário na Argentina no período de 2003 ao 2014. Discute-se com outros trabalhos acadêmicos que sustentam que aquele período coincide com um aumento nos níveis de privatização educativa. Mostra-se, a partir do cruzamento de dados educativos, de rendimento educacional e populacional, que a evolução da matrícula privada não acelera seu ritmo e expansão segue o ciclo econômico. Por sua parte, ensina-se como a matrícula estatal se vê condicionada pela evolução dos indicadores de rendimento interno e a política educativa. No nível secundário, conclui-se que a tendência se corresponde com uma maior estatização e fragmentação do nível. Sustenta-se como hipótese que os sub-registros populacionais e as melhoras no rendimento interno escola impedem ver a maior estatização do sistema educativo argentino e as mudanças que operam nas distintas modalidades educativas. Afirma-se que estas mudanças têm relação com as transformações e a crise orgânica da sociedade em um plano geral. Desde uma perspectiva teórica materialista, entende-se a dinâmica escolar atravessada pelas mudanças nas relações sociais de produção, situação que explica a crescente degradação e estatização escolar.

Palavras-chave: sistema educativo, privatização, tendências, estatização. 


\section{INTRODUCCIÓN}

Este artículo reflexiona sobre las tendencias que afectan a la educación argentina en el siglo XXI. El debate local centra su atención en el nivel de privatización del sistema educativo como una de las características que irrumpe en la segunda mitad del siglo XX y logra consolidarse definitivamente. Más recientemente, una serie de trabajos sostiene que pos-2003 se produciría una "segunda oleada" de privatización. En ese sentido, hace décadas especialistas, investigadores, miembros de organizaciones sindicales y políticas debaten sobre el peso de la educación privada como un problema crucial. Dada la magnitud de la literatura específica, no podemos recomponer el estado del debate completo. Solo se mencionan los trabajos canónicos y los que abordan el periodo reciente objeto de este estudio. En este artículo, definimos privatización como aquellos servicios educativos reconocidos por el Estado como tal, administrados por agentes privados, lo que algunos llaman esquemas de "contratación externa" o privatización exógena (Ball y Youdell, 2007, p. 25). ${ }^{1}$ Se

1 En la Argentina, no proliferaron esquemas de escuelas charter 0 vouchers, lo que no implica que no existan escuelas con "incentivos fiscales". Para el caso argentino, los subsidios son transferencia de recursos del Estado argentino a las escuelas privadas. Más adelante, nos referimos a la magnitud de subsidios transferidos a las escuelas de gestión privada. La definición de privatización exógena de Ball y Youdell (2007) - tengan o no financiamiento por parte del Estado - incluye las formas de contratación de servicios complementarios como limpieza y comedor, esto es, lo que otros autores conciben como privatización "endógena". Aquí nos limitamos a la propiedad de los establecimientos y a la forma en la que estos son contabilizados por la Dirección Nacional de Información y Estadística Educativa (Diniee) dependiente del Ministerio de Educación Nacional. Recordamos que, en términos de Ball y Youdell, la privatización endógena implica la introducción de "normas, valores y lógicas del sector privado en los sistemas educativos", lo que otros autores denominan management o mercantilización. Posteriormente, Ball (2014) sostuvo que "el desarrollo de nuevos tipos de negocios educativos globales como las novedosas formas de comercio de la limita la discusión a la cantidad de establecimientos de propiedad privada y, puntualmente, a la cantidad de alumnos matriculados en ellos, tal como son registrados por la Dirección Nacional de Información y Estadística Educativa (Diniee). Se establece como hipótesis de trabajo que el supuesto crecimiento de la privatización pos-2003 no es más que una "ilusión estadística" que encubre un fenómeno poco conocido: la mejora compulsiva de los indicadores de rendimiento interno, el cambio en la composición histórica de las modalidades educativas y una mayor estatización de la matrícula bajo nuevas formas que responden a las tendencias a la agudización de la crisis orgánica de la sociedad argentina.

Para el caso argentino, el consenso sobre la privatización alcanza a la mayoría de los investigadores y las discrepancias se ubican sobre aspectos puntuales. Acuerdan que la privatización avanzaría desde mediados de la década de los cincuenta, es decir, en sintonía con los planteamientos de Friedman (1997), el ocaso del estado de bienestar y el predominio de la agenda internacional. ${ }^{2}$ Los desacuerdos alcanzan otros aspectos, por ejemplo, la causa subyacente al fenómeno.

economía educativa, trascienden la división educación pública/educación privada, y transforman todos los servicios de la educación en mercancías que pueden comercializarse" (p. 3). Sin embargo, los ejemplos que el mismo autor proporciona niegan el impacto de ese mercado. Para el concepto de mercantilización, véase Whitty y Power (2000), quienes observan el fenómeno para el Reino Unido, los Estados Unidos, Australia y Nueva Zelanda.

2 Sintéticamente, la idea de rodear al Estado de privados lo tornaría más eficiente. Valga de ejemplo la siguiente cita: "Creo que la única manera de crear una mejora sustancial en nuestro sistema educativo es a través de la privatización, hasta el punto en que una fracción sustantiva de todos los servicios educativos sea provista por empresas privadas [...] El problema es cómo llegar desde aquí hasta allí. Los vouchers no son un fin en sí mismos, son un medio para hacer la transición desde un sistema estatal a uno de mercado" (Friedman, 1997, p. 343). 
Algunos autores consideran que la privatización consecuencia de la descentralización educativa sería promovida por el llamado modelo neoliberal, tal como indican Filmus (1997, 2000), Paviglianitti (1995), Puiggrós (1997, 2003) y Torres (2008). Otros agregan que lo específico residiría en la imposición de lógicas gerencialistas impuestas por el llamado Consenso de Washington en la escuela estatal en la que la equiparación de la educación de gestión privada y estatal como de gestión pública "contribuyó a definir un escenario favorable a los procesos de privatización en el terreno educativo" (Feldefber, 2009, p. 35), sobre todo, en la educación superior. Para el resto de los niveles, se verificaría la introducción de lógicas de "cuasimercado" a través de mecanismos de diversificación de la oferta escolar y la promoción de la libertad de elección (Feldfeber, 2006; Feldfeber y Gluz, 2011). Los trabajos producidos en la Confederación de Trabajadores de la Educación de la República Argentina (Consejo Latinoamericano de Ciencias Sociales [Clacso], 2005) también ligan el paradigma neoliberal con la imposición de políticas privatizadoras. Según Tiramonti (1997), la avanzada neoliberal alteró "el imaginario social del pueblo argentino", que pasó de entender el financiamiento de la educación como un derecho humano y un deber del Estado a un derecho a adquirirse por competencia de recursos financieros bajo esquemas de evaluación del rendimiento y con recursos extraordinarios asociados a proyectos educativos especiales. Por su parte, Vior y Rodríguez (2012) entienden que el proceso de privatización en Argentina responde al "modo en que se fueron resolviendo las demandas concretas de los grupos, fracciones o clases a lo largo de su historia" (p. 92). Discuten que la privatización se vincule únicamente con la existencia de regímenes dictatoriales en la región y en el país. Las autoras entienden que la política de subsidios al sector privado iniciadas por el peronismo (1943-1955) permitió ampliar la oferta educativa y cumplir compromisos adquiridos con la Iglesia, principal proveedor de educación privada. Así, las tasas de crecimiento de la matrícula privada serían más altas que las estatales, proceso que en el plano legal habría tenido un embate decisivo en la década de los noventa tras lo cual se consolidó el proceso privatizador. En el plano sindical docente, corrientes ligadas a la izquierda entienden que la privatización educativa no es más que una manifestación más de la crisis capitalista, en la que "el capital" buscaba convertir espacios improductivos en nuevas fuentes de ganancias (Marrone, 2014; Tribuna Docente, 2005). Otros colocan el eje sobre el aporte estatal para la educación privada y sugieren que el periodo de 1950 a 1990 debe entenderse como de crecimiento de la educación privada, pero en un esquema de "publificación", esto es, sostenida legal y materialmente por el Estado mismo (Gamallo, 2015). Para el periodo de 2003 a 2010, Gamallo observa también ascenso absoluto de la educación privada en detrimento de la pública, ya que "el sector privado se presenta como el componente dinámico" (p. 65).

Otros especialistas matizan el nivel alcanzado por la educación de gestión privada, pero entienden igual que sus niveles son altos. Esa serie de trabajos busca explicar la preferencia de las familias de ingresos medios por los circuitos privados, tal como aportan los trabajos de Narodowski y Andrada (2001), Narodowski y Nores (2000), Narodowski, Moschetti y Gottau (2017), 
entre otros. Esa serie de estudios entienden que operan en el sistema educativo formas de privatización interna dentro de un "cuasimonopolio estatal"; otros que se trata de formas de privatización endógena (Marrone, 2014).

Recientemente, se advertía sobre el aumento del dinamismo de la gestión privada en los últimos años, fundamentalmente en el nivel primario. Mariano Narodowski publicó, junto con Mauro Moschetti, un trabajo en que advertían sobre la expansión de la educación privada bajo el llamado kirchnerismo. ${ }^{3}$ Los autores consideraban que, desde 2003, se produjo un hecho inédito en la historia de la educación: la fuga absoluta de alumnos del circuito público al privado. Por un lado, la matrícula privada crecería a gran ritmo y con mayor dinamismo, a la par se produciría una contracción - relativa y absoluta - de la matrícula pública. Esas tendencias operarían en todas las provincias, con excepción de Santa Cruz. El crecimiento privado alcanzaría niveles significativos o críticos en dieciséis provincias. Por eso, los autores concluyen que la privatización del sistema avanza en la llamada etapa posneoliberal (Narodowski, Moschetti y Gottau, 2017). En sintonía con trabajos anteriores, se preguntan por qué las familias argentinas dejaron de elegir la educación pública. Responden que la mala e ineficiente gestión del Estado impulsaría la salida y, a su vez, el mismo Estado provocaría ese traspaso. Los déficits en infraestructura escolar y la escasez de recursos impulsarían políticas de corto plazo; por eso, se subvenciona el

3 Nombre que recibe la corriente política que se corresponde con las presidencias del matrimonio Kirchner. Néstor Kirchner (2003-2007) y las dos presidencias de Cristina Fernández de Kirchner (2007-2011 y 2011-2015). sector privado ya existente, y así se abaratan las cuotas de la educación privada. Por eso, entienden que forma parte de la lógica de "cuasimonopolio" estatal promoviendo la gestión privada (Narodowski, 2008). Narodowski y su equipo concluyeron que existía un sistema de incentivos estatales que favorecía el crecimiento de la educación privada al hallar correlación entre el aumento de los subsidios y el crecimiento de la matrícula privada en el periodo de referencia (Narodoski y Moschetti, 2015a, 2015b). El Estado resolvería así los problemas de cobertura estructural a corto plazo y liberaría recursos para focalizarlos en los sectores más postergados.

Otros autores añadieron más factores explicativos como los bajos rendimientos en pruebas estandarizadas de la escuela estatal y la búsqueda de un mínimo de calidad y continuidad escolar en la privada (Guadagni, 2013), argumento similar a Tenfi y Grimson (2014). Rivas (2015), por su parte, entiende que los conflictos con los sindicatos y la reducción de los días de clase explican la tendencia reciente a la privatización. A partir de datos empíricos entre ciclo de huelgas y aumento de la matrícula, Narodowski, Moschetti y Alegre (2016) concluyen que las curvas de crecimiento de huelgas y privatización no parecen guardar relación concluyente.

En suma, la mayoría de la bibliografía académica y también los posicionamientos político-sindicales consideran que la historia educativa de la segunda mitad del siglo $X X$ a nuestros días es la historia de la privatización. El paradigma neoliberal triunfante, la búsqueda de superar la crisis económica y fiscal pos-Estado de bienestar, las búsquedas individuales de las familias de calidad, 
continuidad educativa o prestigio, las tendencias inexorables del capitalismo son algunas de las explicaciones en boga. Más aún, una nueva camada de trabajos plantea que pos-2003 la privatización del sistema educativo aumentó y generó un fenómeno novedoso: el éxodo absoluto de alumnos del circuito estatal al privado. Incluso, cuando el problema se puso en el tapete en 2014, los funcionarios educativos reconocieron el fenómeno y lo adujeron a la idiosincrasia de la población (mayormente católica), a la bonaza económica y a la disminución de la población en edad escolar dada la caída de la natalidad (La Nación, 2014). Ya hemos mostrado en otro lugar que en Argentina no puede hablarse de un proceso de privatización durante la segunda mitad del siglo XX dada la baja participación del sector privado (De Luca, 2017a). En este artículo, se examina esa supuesta "segunda oleada" de privatización. Como se anticipó, considera que se trata de una "ilusión" estadística que impide comprender las tendencias más profundas que operan en el sistema educativo argentino: degradación, nuevas formas de estatización y cambios en las modalidades educativas. Para eso, se examina, junto con la evolución de la matrícula escolar, el crecimiento poblacional y los indicadores de rendimiento interno del sistema educativo. A juicio de los autores, la construcción del relato y el deterioro de las estadísticas (Sartelli, 2018) impidió comprender las tendencias reales a nivel educativo.

\section{MARCo TEÓRICO Y METODOLOGÍA}

La mayoría de los estudios desprende la dinámica escolar de la política educativa e infiere la existencia de dos paradigmas: el estado docente y su ocaso con la emergencia de políticas de privatización. Este artículo sostiene que no es posible pensar la dinámica escolar escindida de la dinámica social (Sartelli, 2013). La escuela argentina forma parte de una sociedad específica (la sociedad capitalista) y es ella la que imprime las "tendencias" más generales al sistema educativo. Para el periodo que se analiza, la crisis orgánica de la sociedad argentina, la expulsión de población por la misma dinámica de las relaciones sociales de producción y la profundización del régimen de gran industria determinan la tendencia general. Forman parte de los presupuestos teóricos de los autores que, si hoy la escuela argentina alcanza niveles de desigualdad, de fragmentación, de segmentación y de decadencia de una magnitud pocas veces antes vista, es expresión de la adecuación de la dinámica escolar a la tendencia a la degradación de las calificaciones necesarias de la fuerza de trabajo impuestas por el capitalismo. Los trabajadores menos calificados - a través de la simplificación de sus tareas - requieren menos educación, fenómeno que cuesta ser percibido dadas las tendencias crecientes a la extensión de la escolaridad. Pero ese movimiento de simplificación de tareas, de reemplazo de trabajo vivo por muerto (humano por máquinas), se impone a través de múltiples mediaciones y tiene sus propias causas contrarrestantes. Algunos pocos requerirán mayor calificación. El resultado: la polarización de conocimientos. Por un lado, un puñado de trabajadores hipercalificados (desde los creadores de las máquinas, hasta quienes deberán ser calificados para operarlas); por otro, la amplia mayoría de trabajadores descalificados, desespecializados, algunos meros apéndices y otros ya "inservibles", el amplio ejército de reserva 
de los desocupados (Sartelli y Kabat, 2014). Braverman (1975) es quien, siguiendo las ideas de Marx, desarrolló ese aporte en su ya clásico Trabajo y capital monopolista. La descentralización del sistema educativo, y no la privatización, cuya primera expresión en materia curricular apareció en escena en la década de los sesenta, no hizo más que profundizarse como respuesta a esa polarización (De Luca, 2011, 2017a). En varios trabajos, dimos cuenta de esas tendencias estructuradoras de la dinámica social y su correlato en el ámbito escolar (Kabat, 2005, 2007; Sartelli, 2007, 2011, 2013).

Este artículo discute con los trabajos que conciben una profundización de la privatización en el siglo XXI, porque no problematizan las inconsistencias de las estadísticas oficiales en materia educativa. El ocultamiento estadístico y la negación del problema de la degradación escolar responde a la forma en la cual el régimen kirchnerista - que asciende al poder en un periodo de crisis - se legitima: la construcción ideológica sobre su lugar histórico en eso que se popularizó bajo el nombre de "relato" (Sartelli, 2017). La supuesta "reinvención de la escuela", la mejora de la calidad y una visión pedagógica inclusiva aparecieron como los ejes principales $y$, para alcanzarlos, se debía desandar la herencia anterior. Los logros de la ley federal sancionada en la década de los noventa no parecían muy notables, al margen de amontonar alumnos. Hacia 2000, el 43,9 \% de los alumnos argentinos obtenían resultados iguales o debajo de 1 en comprensión lectora, siendo 5 es el más elevado. ¿Qué significaba ello? Que prácticamente no sabían leer (Fondo de las Naciones Unidas para la Infancia [Unicef], 2003). El kirchnerismo supo leer la temperatura ambiente. Para desmovilizar a la sociedad pos-2001 debía tomar algunas de sus consignas, aunque fuera para hacer algo muy parecido a sus antecesores. Supo ver que para desmovilizar a la clase media (pequeño-burguesía) debía tocar algunas de sus fibras más sensibles. La educación fue (y es) una de sus demandas centrales. También de la clase obrera. Así, el kirchnerismo inició su "transformación". Prometió mejorar la calidad. Prometió unificar el sistema. Prometió mejorar las condiciones de trabajo docente. Bregó por la inclusión pero su escuela siguió siendo tan clasista y fragmentada como la pasada. Pero sí se encargó de ocultar su propio fracaso y construyó un "relato".

En este artículo, se trabaja con estadística educativa, datos poblacionales, condiciones de vida, indicadores de rendimiento interno del sistema escolar e información periodística. Para la evolución de la matrícula escolar, nos valemos de los datos de los relevamientos anuales elaborados por la Dirección Nacional de Información y Estadística Educativa (Diniee) e información especial sobre rendimiento interno suministrada a los autores, los censos nacionales de población 2001 y 2010, las estimaciones y proyecciones de población elaboradas también por el Instituto Nacional de Estadísticas y Censos (Indec), datos poblacionales provenientes de las estadísticas vitales del Ministerio de Salud, informes de los técnicos. Comparamos la evolución de la población según los censos y las estadísticas vitales. A partir de las inconsistencias entre ambos, recurrimos a los informes técnicos sobre los censos y las proyecciones poblacionales. A partir de esos datos, y siguiendo los cálculos del Indec, arribamos a la población en edad escolar 
estimada. Comparamos esos datos con los poblacionales y las estadísticas educativas. Por último, procedimos a realizar cálculos sobre los cambios en la composición de la matrícula de acuerdo con los indicadores de rendimiento interno del sistema escolar y los subregistros de la población. A partir de esos insumos, se extraen las conclusiones que aquí se presentan.

\section{LA MATRÍCULA ESCOLAR DEL NIVEL PRIMARIO}

La discusión sobre la evolución reciente del sistema privado y público se concentró en el nivel primario. De los números, llamaba la atención que la matrícula total (gestión pública más privada) se redujera, lo que debería ir acompañado de una reducción de la población total o de una caída en las tasas de escolarización. Sin embargo, los datos oficiales no abonaban ninguna de las dos situaciones. La caída poblacional de los niños de 5 años (población base para justificar el descenso de matrícula) es registrada por el censo de 2010, censo que tuvo serias y graves irregularidades: cartografías viejas, lo que derivó en un subregistro censal y metodología incompleta para la corrección y el vuelco de la información. Al comparar los resultados del censo con las estadísticas vitales del Ministerio de Salud, pudimos ver que la caída poblacional no se condecía con los datos y más bien ubicaba los valores del censo de 2010 en un subregistro de la población de 5 años en promedio de poco más del 9 \%. Según la información oficial, la omisión del censo sería de apenas el 0,79\% ( $L a$ Voz, 2011). Sin embargo, los técnicos desplazados por el Indec - en el proceso de intervención sobre el organismo iniciado por el kirchnerismo- señalaron que no fueron censadas cerca de cuatro millones de personas:

Hubo innumerables testimonios de personas que no fueron censadas. La decisión de las autoridades de la intervención de no realizar la encuesta pos-censal, que es la que corrobora la cobertura efectiva que tuvo el Censo, fortalece aún más la sospecha sobre la veracidad de los resultados. Llamativamente, los resultados globales tienden a coincidir con las proyecciones de población realizadas sobre los datos del Censo 2001. (Junta Interna ATE-Indec, 2010a, párrafo 4)

Además, "el total de población dado a conocer no coincide con el que se manejó durante los procesos de la información [...] los faltantes fueron disimulados" (Almeida y Pok, 2014, p. 90). Se denunció también que los métodos de relevamiento, listado y conteo de viviendas, el vuelco de la información y la segmentación, así como la preparación de la cartografía, se realizaron en forma tardía. Inclusive fueron corregidas por las direcciones de estadística $a$ posteriori de su diseño. Como consecuencia, se utilizó la cartografía de conteo de viviendas de 2001, lo que produjo numerosos desfasajes con la situación real del territorio (Junta Interna ATE-Indec, 2010b, p. 13). Una situación similar fue denunciada para el censo experimental, realizado en Chivilcoy: mientras el relevamiento censal contó 47000 hab., el censo municipal registraba 73855 hab. ("Proyecto de Resolución La Honorable Cámara de Diputados". La supuesta disminución de la población de 5 años no se condice tampoco con los datos de las estadísticas vitales publicados por el Ministerio de Salud en el periodo de 2000 a 
2013. La cantidad de nacimientos creció tanto como el crecimiento de la población total declarada por el Indec en el censo de 2010: el $10,6 \%$. No puede argumentarse que hubo menos nacidos en ese periodo y por eso la población escolar cae. El censo de 2010 registró 676130 niños de 5 años. Sin embargo, cinco años antes, en 2005, nacieron 712220 personas y durante 2006 fallecieron 8986 menores de un año, lo que daría una población total de 703 234. La población real que el censo debería haber registrado tendría que ser, como mínimo, un $4 \%$ más. Aun así, la cifra construida sigue siendo menor a la registrada en 2001 sin modificarse prácticamente la paridez media. Un trabajo realizado por la Asociación Civil Educación para Todos examinó los desfasajes entre los censos y las estadísticas vitales del Ministerio de Salud. Se concluyó que, si bien las tendencias eran congruentes, las diferencias entre la población censada y la registrada por el Ministerio de Salud oscilaba entre el 2 y el $10 \%$ (Unicef, s. f., p. 24). A cifras similares se arriba tomando las proyecciones poblacionales realizadas por el Indec en 2004 y ajustando los valores poblacionales con la población en edad escolar, tal como dispone los métodos de proyección de la Diniee.

Este resulta un punto de partida importante dado que una de las explicaciones para la caída de la matrícula estatal y total residía en la disminución de la población de 5 años. Como vemos, eso no se condice al cruzar distintos datos estadísticos. Veamos, a continuación, cuál fue la evolución de la población escolar del nivel primario según los datos educativos y cómo puede repensarse el problema.

Según los datos oficiales de la Diniee, en el nivel primario común, la matrícula estatal pasaba de 3747747 en 2003 a 3334259 en 2014, una contracción total de 413488 alumnos. Por su parte, la evolución del sector privado era ascendente: de 970863 a 1216664 alumnos, en el mismo periodo (Dirección Nacional de Información y Evaluación de la Calidad Educativa [Diniece], 2003, 2014). Así la matrícula total de la primaria común caía 167687 alumnos en once años. Esa caída afectaba por igual a todas las provincias con excepción de San Juan, Santa Cruz y Tierra del Fuego. Nótese, además, que la caída de la matrícula pública no aparece compensada por el crecimiento de la privada, lo que sería de esperar si se produjera el mentado "traspaso". Si bien en la década de los noventa la matrícula primaria crecía a menor ritmo que en décadas anteriores, nunca había dejado de aumentar en términos totales. Tampoco se encuentra, en otros momentos, una contracción de la matrícula pública. Esclarecer este punto resultaba crucial para determinar si el sistema educativo avanza hacia su privatización.

En primer lugar, como se mostró en el estado de la cuestión, la mayoría de los trabajos sostiene que, a partir de 2003, se produjo una aceleración en el crecimiento de la matrícula privada. Pero una mirada de largo plazo desmiente su presunto dinamismo, tal como evidencia la figura 1 .

Como muestra la figura 1, el crecimiento interanual de los alumnos del sector privado se encuentra en los mismos rangos que desde la década de los sesenta, es decir, cuando la modalidad tenía un volumen menor. Por ende, solo se puede concluir que el dinamismo es mayor en el pasado que en la fase actual. Al analizar la matriculación a primer grado de la escuela primaria, se encuentra 


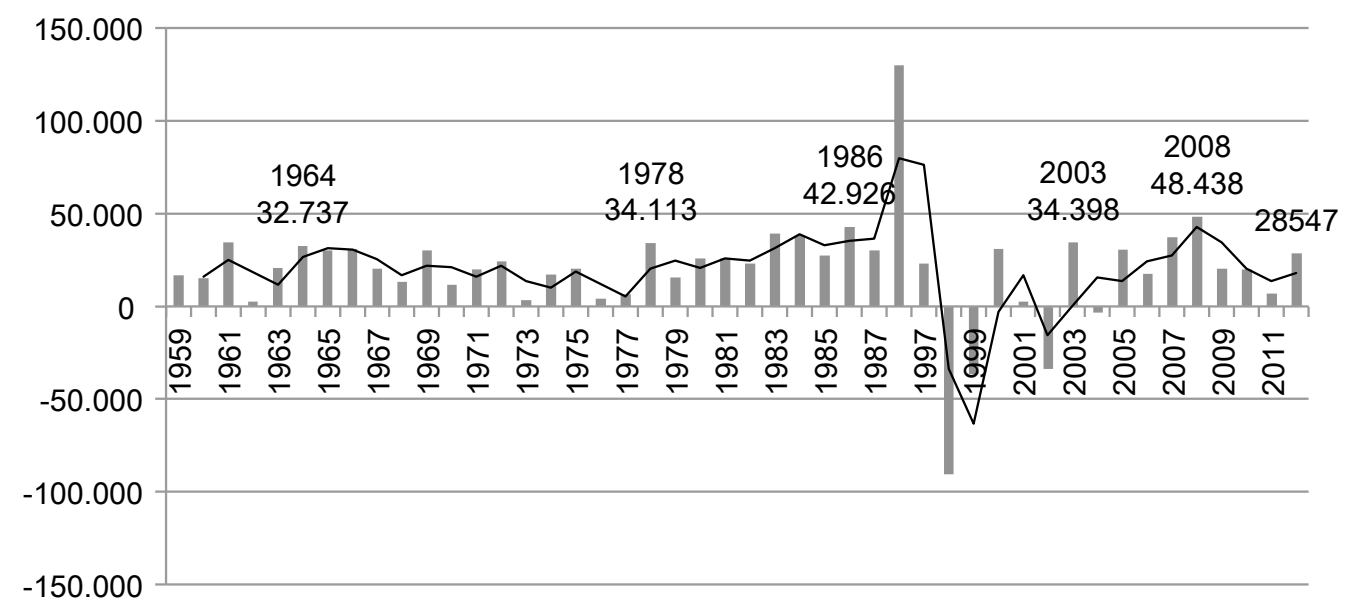

Adición de alumnos gestión privada

— 2 per. media móvil (Adición de alumnos gestión privada)

Figura 1. Argentina. Nivel primario de educación. Modalidad común. Gestión privada.

Aumento interanual de la matrícula. Total del país, 1959-1987 y 1997-2013.

Fuente: Elaboración propia según La educación en cifras, varios números y Estadísticas de la educación, 1959-1986 y Anuario Estadístico Educativo de 1997 a 2013, Diniece.

que, entre 1996 y 2014, se registran evoluciones disímiles. Hasta 1998 ambas gestiones crecen, el sector estatal pasa de 655406 alumnos en primer grado a 699056 mientras el sector privado se mueve de 163229 alumnos a 171 940. Hasta 2002, ambas gestiones descienden (entre 1999 y 2000) y estabilizan su matrícula golpeadas por la crisis política, económica y social que se conoce como Argentinazo (Sartelli, 2007), las jornadas del 19 al 20 de diciembre en las que renuncia a la Presidencia Fernando de la Rúa y se abre una crisis política sin precedentes. A partir de ese momento, ambas gestiones sufren evoluciones diferentes. Mientras la matrícula estatal de primer grado desciende en forma sostenida y pasa de 674884 alumnos en 2003 a 533789 en 2014, la matriculación privada en primer grado asciende de 172453 alumnos a 212415 en 2014. Esta evolución debería llamar la atención, porque implica que desde 2003 el $100 \%$ de la matrícula se habría volcado al sector privado, aun los sectores más empobrecidos y pauperizados de la población, en un país donde la pobreza alcanzó cifras cercanas al $36 \%$ (Perfil, 2014), el desempleo al 28 \% (Infobae, 2015) y el empleo informal y precario de más de un tercio de los trabajadores. Resulta evidente, al constatar esas cifras como problema general. Inclusive en las provincias más pobres del país (región noreste y noroeste). ${ }^{4}$ donde la pobreza, el desempleo y la informalidad son más agudos, se requería indagar otros indicadores. Por lo menos a modo

4 En Chaco, la matriculación a primer grado del sector estatal en 2014 se reduce al $63 \%$ de los alumnos matriculados en 2003 , al igual que en Corrientes; en Formosa al $60 \%$; en Jujuy al $73 \%$; en Misiones al $61 \%$; en Salta al $75 \%$, al igual que en Tucumán; en Santiago del Estero, al $59 \%$. 
de hipótesis, considerar cómo los subregistros poblacionales se traducen en ausencia de acceso al sistema escolar y si algún factor de la dinámica educativa explicaba la caída absoluta de la matrícula estatal.

En este sentido, este artículo aporta elementos que no fueron considerados antes en los análisis sobre el tema: la reducción de la matrícula pública se corresponde con una fuerte contracción de la repitencia y de la deserción. Esta mejora fue impulsada en forma "compulsiva" por la política educativa cuando se sostuvo que se debía desarrollar una escuela "inclusiva" en la que ni la repitencia ni la deserción eran válidas pedagógicamente hablando. Por eso, los funcionarios sugerían imaginar nuevas estrategias de trabajo para los "nuevos" sujetos que llegaban a la escuela, en particular refiriéndose a la extensión de la obligatoriedad, en la década de los noventa la EGB 3 y pos-2006 el secundario completo (Resolución CFE № 174/12).

En efecto, la reducción de la matrícula pública se correspondió con una fuerte contracción de la repitencia y de la deserción a partir de 2003, producto, primero, de la aplicación del programa "Todos pueden aprender" coordinado por la Unicef y, luego, toda una serie de programas subsidiarios similares. El programa de la Unicef buscaba reducir los niveles de repitencia de la población con "alto riesgo social", en particular en el primer grado, en que la repitencia alcanzaba casi el $10 \%$. En 2000, la repitencia de primer grado era del 10,38 \% (Ministerio de Educación, 2012, p. 5). En la primera etapa, el programa se desarrolló en las provincias de Tucumán, Misiones, Chaco y Jujuy (Unicef, 2003, p. 21; 2005, p. 10) con 11000 niños de primer grado a los que se les entregaron además kits escolares con material didáctico. En 2005, el programa se amplió a 23000 participantes; en 2006, a 30 000. Entre 2003 y 2007, la repitencia de primer grado disminuyó el 9,3\% porque pasó del 13,7 \% al 4,4\% (Unicef, 2007, p. 15). En 2007, el programa se amplió a más provincias: Entre Ríos, Formosa, San Juan, San Luis y Santa Fe, alcanzando a 90000 alumnos. En ese año, se presentaron ante al Congreso de la Nación los resultados del programa que pasó a ser política pública (La Gaceta, 2007). En 2012, se anunció la promoción automática entre primer y segundo grado, con lo cual la repitencia (que en algunas provincias alcanzaba más del $20 \%$ ) fue erradicada de cuajo (Resolución CFE № 174/12).

Esa serie de medidas y sugerencias tuvieron gran impacto en la escuela primaria. En el sector público, la tasa de repitentes pasó del 6,50 al 2,90\% en el periodo de 2003 a 2013, lo que implica una caída del 4,20\%. En el sector privado, la repitencia también disminuyó, aunque sus valores son bajos respecto de su par estatal: cae el 0,70\% al pasar del 1,40 \% en 2003 al 0,70 \% en 2013. El dispar rendimiento del circuito público respecto del privado se profundiza en la evolución de la sobreedad. El sector público registraba una sobreedad del 27,06 \% en 2003. Sin embargo, diez años más tarde, el indicador se ubicó por debajo de los veinte puntos: el 19,86 \%. La disminución de la tasa de sobreedad en diez años superó los siete puntos $(7,20 \%)$ en el sector estatal. En el circuito privado, la sobreedad cayó 2,86 puntos al pasar del 6,42 al 3,57 \% (Diniece, 2015). Si bien ambas gestiones siguen la misma tendencia, en el sector público los indicadores son cinco o seis veces más altos que los del sector privado. 
Por ello, la tasa de mejora incide más en el circuito estatal, no solo por su volumen, sino también por su mayor matrícula. Por su parte, las tasas de promoción efectiva y de abandono interanual siguen trayectorias divergentes en cada gestión: mientras en el Estado los indicadores mejoraron, en el privado empeoraron. ${ }^{5}$

Resulta importante calcular, entonces, cuál es el volumen de alumnos a los que afectan esas cifras. Al hacerlo, se halla que 525000 alumnos que antes repetían o desertaban ahora pasan de grado/año y permanecen en la escuela. ${ }^{6}$ Cae la sobreedad y el abandono producto de la mejora de esos indicadores. Lógicamente, si la tendencia es a la mejora, entonces la matrícula que antes aparecía "abultada" por bajos niveles de promoción efectiva, repitencia y sobreedad, circula ahora más rápidamente por el nivel. El resultado es un efecto de "caída" de la matrícula estatal. Este punto explica también la supuesta caída en la matriculación de primer grado si consideramos que la repitencia es superior ese año respecto de los siguientes y que varios de los programas descriptos iniciaron trabajando sobre el pasaje automático y la eliminación de la repetición en ese grado. No resultaba factible considerar que, en las provincias más pobres de la Argentina, el $100 \%$ de los alumnos se matriculen en escuelas privadas, incluso, en zonas donde

5 La Diniee entregó los datos a pedido de los autores como informe y solo se hallaban disponibles, en ese momento, hasta 2013.

6 La cifra surge de restar la diferencia entre la matrícula registrada por la Diniee para 2003 para el nivel primario común (3 667286 alumnos en todo el país) y la registrada para 2013 (3 365379 alumnos) sumando el índice de mejora de la matrícula en esos años de referencia: el 4,20\% de mejora en la repitencia en el periodo de 2003 a 2013 , el 7,2 \% para la sobreedad, el 5,7 \% para la promoción efectiva y el 1,5\% para el abandono interanual. Así se observa la evolución de la matrícula como si no hubiera estado afectada por la política educativa de promoción automática instaurada en esos años. las escuelas privadas se encuentran emplazadas únicamente en capitales provinciales o departamentos capitales o de gran actividad. Como contraparte, los indicadores en la gestión privada comenzaron a empeorar, y una consecuencia de ese proceso es que su matrícula se "abulta" artificialmente.

Para la comprensión total del problema, hay que sumar otros dos elementos. Por un lado, volver sobre lo dicho acerca de la evolución poblacional. Los extraños números a los que nos referimos en la evolución de la matrícula primaria en regiones como noreste, noroeste y centro abonan la perspectiva de que el subregistro de la población también se manifiesta en materia educativa. Existen capas enteras de la población que no estarían accediendo al sistema educativo, problema que afecta al nivel primario y con más fuerza aún al secundario. Por otro lado, advertimos sobre los cambios en la composición histórica de las modalidades del sistema educativo. En el nivel primario, se hace presente, aun de forma incipiente, un hecho que, como se desarrollará luego, afecta con fuerza al nivel secundario: el pasaje de matrícula de la modalidad tradicional (común) a otras, como la de adultos, que se presentan como vías más rápidas para el acceso al título o la titulación de los "nunca incluidos" en el nivel.

En efecto, al examinar la evolución de la matrícula y el nivel de privatización comparando los números oficiales, eliminando la mejora compulsiva de los indicadores de rendimiento interno - ubicarlos en los niveles de 2003 - y adicionando el promedio de subregistro poblacional, la evolución del nivel primario resulta bien diferente, tal como resume la tabla 1 . 
Tabla 1. Argentina. Nivel primario. Modalidad común. Matrícula del nivel primario por tipo de gestión. Datos Diniece. Matrícula reconstruida mejora indicadores, matrícula reconstruida con subregistro poblacional. Total del país, 2003 y 2013

\begin{tabular}{lccccc}
\hline Datos & Datos Diniee & Datos Diniee & $\begin{array}{c}\text { Matrícula } \\
\text { Reconstruida. } \\
\text { Indicadores de } \\
\text { mejora }\end{array}$ & $\begin{array}{c}\text { Estimación } \\
\text { matrícula } \\
\text { subregistrada al } \\
\mathbf{9} \% \text { Subregistro } \\
\text { poblacional }\end{array}$ & $\begin{array}{c}\text { Matrícula } \\
\text { reconstruida } \\
\text { final }\end{array}$ \\
\hline Año & 2003 & 2013 & 2013 & 2013 & 2013 \\
\hline $\begin{array}{l}\text { Matrícula } \\
\text { estatal }\end{array}$ & 3667286 & 3365379 & 3890378 & 350134 & 4240512 \\
\hline $\begin{array}{l}\text { Matrícula } \\
\text { privada }\end{array}$ & 953630 & 1198112 & 1247834 & 0 & 1247834 \\
\hline
\end{tabular}

Fuente: Elaboración propia según Anuario Estadístico, Diniece, 2003 y 2013 e indicadores de eficiencia interna del sistema, datos proporcionados a los autores por la Diniee Ref. Nota 822/15, entregados a los autores el 3 de julio de 2015 y datos de población.

Así, al incorporar esos indicadores, el crecimiento de la privatización se reduce sustantivamente: pasa del 5,61 al 2,09\%, y en lugar de controlar el $26 \%$ de la matrícula en 2013, cuando en 2003 sus valores eran del $21 \%$, pasa a un $24 \%$. Es decir, si suponemos que un $9 \%$ de la población está subregistrada, también en materia educativa el crecimiento de la gestión privada en diez años se reduce a dos puntos. Según los datos oficiales, de cada 100 alumnos del nivel primario, 74 asisten a escuelas estatales; pero, según nuestros datos, esa cifra asciende a 77 si ponemos en consideración los datos poblacionales y educativos. Quienes suponen que hay un proceso privatizador en marcha, a juicio de los autores de este artículo, pierden de vista la totalidad del problema. Más aún, hasta el momento, venimos trabajando con la modalidad común del nivel primario. La modalidad común constituye el $99 \%$ de la matrícula privada, mientras que para la pública es el $91 \%$. Si en lugar de tomar solo la matrícula de la modalidad común, incorporamos los valores de la educación especial y de adultos, la privatización se estanca en el $21 \%$.
Se concluye que la mejora de los indicadores de rendimiento interno no solo genera una ficción en términos estadísticos (la supuesta privatización), sino que tiene un correlato curricular muy marcado. Mayor promoción efectiva y menor repitencia no fueron acompañados por una mejora en el rendimiento educativo en los test estandarizados nacionales e internacionales. Según el Tercer Estudio Regional Comparativo y Explicativo (Terce), Argentina obtiene los mismos resultados educativos que Perú y muy poco por arriba de Honduras, Ecuador, Panamá y Guatemala (Ganimian, 2014). Un cuarto de los alumnos de tercer grado no alcanzaron resultados mínimos en lectura y matemática. A los 15 años, un tercio no entiende lo que lee y no puede resolver una regla de tres simple. Según los operativos nacionales de evaluación, casi un tercio de los alumnos obtiene resultados bajos en matemáticas, y un cuarto, bajos en lengua, al egresar de la escuela secundaria. Obtienen, incluso, peores resultados que lo que resultaría consistente con la tasa de pobreza. Este cuadro contrasta con la misma historia del 
país cuando Argentina era modelo para el resto de América Latina.

En paralelo, es probable que una parte minoritaria del circuito privado esté recibiendo "nueva matrícula" proveniente de las fracciones más pauperizadas de la pequeña burguesía y de la clase obrera acomodada. Cierta correlación en los indicadores de rendimiento interno apoyaría tal suposición, ya que el sistema privado empieza a "copiar" algunas tendencias históricas del sector público. En diez años, la promoción efectiva de los alumnos cayó del 98,5 al 97,8 \%. En paralelo, el abandono aumentó y pasó del 0,1 al $1,4 \%$. No sería extraño que de profundizarse esa tendencia la matrícula privada empiece a evidenciar los síntomas del rezago.

Además, la figura 1 permite observar que el crecimiento de la matrícula privada se encuentra en relación con la evolución del ciclo económico del país. La matrícula se contrae al color de la crisis económico-política y social de fines de la década de los noventa, se recupera y sigue una curva ascendente hasta 2008 (año de crisis económica y de reducción de la renta agraria), curva que se retrae luego con excepción de 2012 y 2014. Por, eso, el crecimiento de la gestión privada debe ser visto como una expansión condicionada. Pero existe un segundo elemento que grafica aún más la situación endeble del sector: la mayor parte de la matrícula y de las escuelas privadas se encuentran subsidiadas. Según los datos del Ministerio de Educación de la Nación, el 69 \% de las escuelas y el $75 \%$ de los alumnos de escuelas privadas recibían subsidios estatales en 2010 (Diniece, 2013). Buena parte del crecimiento del sector no podría llevarse a cabo sin la intervención de las arcas estatales. Los subsidios, al abaratar la cuota, facilitan el acceso de algunas fracciones de la clase obrera acomodada a la educación privada. Pero su permanencia pareciera estar condicionada, entonces, por la bonanza económica general y por los favores del arca pública, en particular. Entonces, no solo la educación privada no crece, sino que su existencia depende férreamente de la tutela estatal.

\section{LA MATRÍCULA ESCOLAR DEL NIVEL SECUNDARIO}

Los datos oficiales de la Diniee muestran que la matrícula estatal pasó de 2467598 a $2786329,{ }^{7}$ mientras que el sector privado lo hizo de 924260 a 110 138; ambas gestiones crecieron. La participación de cada sector pasó del 73-27 al 72-28 \%, es decir, la privatización aumentaría apenas un punto, en once años. El predominio estatal resulta indiscutible con un control sobre el 73-72\% de la matrícula. Pero ese crecimiento del sector privado es nulo si incorporamos al análisis las restantes modalidades del nivel: adultos (que incluye formación profesional), especial y técnico-profesional. En esas modalidades, la presencia del Estado es total.

En la educación de adultos, el Estado contaba con una matrícula de 781180 alumnos hacia 2003. ${ }^{8}$ Para 2014, la cifra asciende a 933 450. ${ }^{9}$ Por su parte, la educación privada conforma apenas la décima parte: para 2003, su matrícula era de 73950 y para 2014 de 113 039. La participación público-privado,

\footnotetext{
7 Se incorporaron los alumnos de la modalidad artística.

8 La cifra se divide en 445594 en educación de adultos (centros educativos de nivel secundario [CENS] y bachilleratos) y 335586 en formación profesional.

9 La composición es de 489710 para adultos y 443740 para formación profesional.
} 
entre 2003 y 2014, pasó del 91-9 al 89-11 \%. En lo que se refiere al movimiento particular, la presencia estatal pasó del 93 al 91 \% en la modalidad secundaria de adultos y en la formación profesional del 89 al 88 \%. Sí debe destacarse que la matrícula de la modalidad adultos estatal sufre oscilaciones: la matrícula se contrae en el periodo de 2004 a 2008, recupera valores ligeramente superiores a los de 2004 en 2011 y luego se estanca. Este estancamiento coincide con el lanzamiento de programas de alfabetización y de terminalidad, primero, y de secundario acelerado, después, por parte del Estado, que compiten con la oferta tradicional de adultos.

En lo que se refiere a la modalidad especial del secundario, el movimiento es una clara estatización. En 2003, el Estado contaba con 1143 alumnos, mientras que el sector privado registraba una matrícula de 1330; para 2014, los números son 10675 y 4109, respectivamente. La participación estatal pasó del 46 al $72 \%$.

Aunque a menor escala, algo similar ocurre con la educación técnico-profesional. Aquí también la presencia estatal es masiva: representa casi el $90 \%$ de la modalidad. Si para 2003 el Estado contaba con una matrícula de 165835 alumnos, para 2014 su matrícula asciende a 563 437. Su participación sobre el nivel aumentó poco menos de un punto y pasó de controlar el 89,65 \% del nivel a hacerlo sobre el 90,40 \% de la modalidad. Por su parte, en el mismo periodo, el sector privado pasó de una matrícula de 19155 alumnos a otra de 59894 .

Tal como evidencian los mismos números de la Diniee, no puede hablarse en el nivel medio de ningún proceso de privatización. Más aún, si se agregan todas las modalidades, la participación público-privada no se altera entre 2003 y 2014. Vale aquí también lo relativo a los subsidios ya señalado para el nivel primario.

Resulta importante dilucidar la evolución en el nivel secundario. La Ley de Educación Nacional (Ley № 26.206) aumentó la obligatoriedad escolar de 9 a 12 años. Ese aumento en la obligatoriedad no se tradujo, a diferencia de lo ocurrido en el nivel primario, con una mejora en los indicadores de rendimiento interno: o bien se agravaron, o bien permanecieron estancados. En 2003 y 2013, la tasa de sobreedad era de un 40,5\% y, en el periodo de 2010 a 2011, alcanzó el valor más elevado con un 46,4\%. La tasa de promoción efectiva siguió dos ciclos. Entre 2003 y 2006, la promoción pasó del 75,8 al 71,9 \%. A partir de 2007, inicia una fase de acenso en torno, más o menos, al 75 \%. Ello implica la existencia de procesos de discontinuidad escolar, de repitencia o de deserción, en tanto la promoción efectiva refleja la cantidad de alumnos que se matriculan en un año inmediatamente superior al año de cursada sumado a los datos de sobreedad que implica que los alumnos ingresan con más años de la edad escolar teórica (ideal). Algo similar ocurre con los indicadores de abandono y de repitencia. Ambas tasas siguen una trayectoria ascendente entre 2003 y 2006 a 2007. En ese ciclo, el abandono interanual pasa del 14,1 al $15,1 \%$ y la repitencia pasa del 10,10 al $13,5 \%$ elevándose al 3,4\% en este último caso. El segundo ciclo se corresponde con una mejora de esos indicadores, mejora que, en general, se extiende hasta 2010-2011 para luego estabilizarse en torno a los valores más bajos alcanzados en ese segundo momento. El abandono desde 2007 cae al $12,2 \%$, valores en los que se estabiliza para 
llegar a 2013, con una tasa de abandono interanual del 12,5\%. Por su parte, desde 2007, la repitencia cae al $12,4 \%$, valores en los que se estabiliza con pequeñas oscilaciones para llegar a 2013 con una tasa del 11,7 \%. Como vemos, en el periodo de 2003 a 2013, el abandono cae, pero lo hace en detrimento de una mayor repitencia en el nivel. La evolución de la matrícula de una posible cohorte educativa refleja también esa realidad. En 2008, la matrícula pública de séptimo grado era de 599 204, mientras que la privada ascendía a 182 571. En 2013, se encontraban en el último año del secundario estatal (doce año de estudio) 256664 alumnos y 144788 en el privado. Así, el nivel de supervivencia en el Estado fue del $43 \%$, mientras que en el sector privado se mantuvo en torno al $80 \%$. El dato empeora si incorporamos el nivel de egreso: en el sector público, solo egresaron 183 800, apenas el $72 \%$ del $43 \%$ que inició los estudios secundarios. Es decir, de cada 10, apenas 3 terminan. En el sector privado, los números son algo mejores: egresaron 118 670, el $82 \%$ de los que llegan al último año; esto es, de cada 10 que inician casi 7 lo terminan.

Si reubicáramos a los desertores de uno y otro lado, y supusiéramos que la matrícula se mantiene constante en los valores de la cohorte inicial, la composición de la matrícula sería del 79-21\%, es decir, se habría estatizado seis puntos. ${ }^{10}$ Puede parecer un ejercicio arbitrario, a fin de sostener nuestra hipótesis que niega la privatización, pero no lo es. Porque hay que preguntarse qué

10 El ejercicio surge de incorporarle a la matrícula reportada por la Diniece para cada año la cantidad de alumnos faltantes de los que empezaron en 2008 a la matrícula existente. Al analizar la evolución de matrícula de cada uno de los años, en el pasaje de octavo a noveno, la matrícula se reduce el $17 \%$, entre noveno y décimo otro $15 \%$, entre décimo y once un $25 \%$, entre once y doce otro $21 \%$ más. De los que llegan a doce, no egresa el $29 \%$ como ya se señaló. ocurre con esos 7 jóvenes que no terminan el secundario común. ¿A dónde van? Antes, en las décadas de los ochenta y noventa, a ningún lado. Pero ahora, con la expansión de la educación de adultos y de los planes de terminalidad, la situación cambia de modo estructural, cambio que no ha sido percibido por los defensores de la tesis de la privatización. Veamos.

La figura 2 muestra la evolución de la matrícula de adultos desde 1996. Tal como puede verse, entre 1997 y 2004, la matrícula casi se duplica, inicia una fase de estancamiento o caída hasta 2009 para recuperarse en un nuevo ciclo de ascenso. En efecto, en el periodo de 2003 a 2014, la matrícula crece el $25 \%$. Al igual que la primaria, la educación secundaria de adultos es masivamente pública, con más del $90 \%$ a cargo del Estado. Entre 2003 y 2014, se incorporaron a escuelas secundarias para adultos 62650 estudiantes y la matrícula total pasó de 476840 a otra de 539 490. De esos, 44116 lo hicieron en el Estado. El proceso fue de la mano de un "rejuvenecimiento" de la matrícula: si en 2008, la franja 20-29 representaba el $38 \%$ del total de alumnos, para 2014 crece al $43 \%$.

Por eso, el circuito de adultos formal es una opción cada vez más elegida por las capas de jóvenes desertores de la escuela "común". Así lo demuestra la nueva composición etaria. La educación de adultos tradicional dirigida a adultos mayores trabajadores hoy se integra en paralelo a la escuela común y recibe cada vez más a jóvenes de clase obrera desertores. En ese recambio, pierde el perfil tradicional que la organizaba. Los jóvenes permanecerán allí unos años. Entran y salen del sistema para luego integrarse a la nueva opción tituladora: "Fines 2", "Adultos 2000”, “Terminá la secundaria”, entre otros. 
Educación Secundaria. Modalidad Adultos. Gestión Estatal. Total del país. Argentina, 1997-2014.

- 2 per. media móvil (Educación Secundaria. Modalidad Adultos. Gestión Estatal. Total del país. Argentina, 1997-2014.)

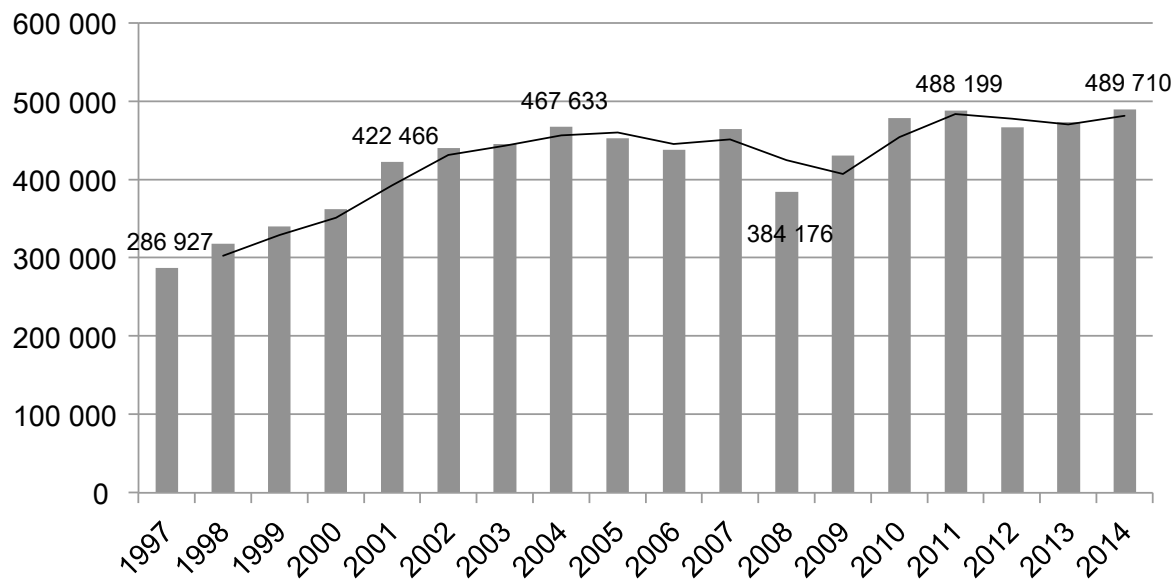

Figura 2. Argentina. Matrícula por año. Nivel secundario de educación. Modalidad adultos. Gestión estatal. Total del país, 1997-2014.

Fuente: Elaboración propia según Relevamiento anual, Diniece 1997-2014.

No podemos desarrollar in extenso este punto. Solo diremos que la evidencia disponible muestra que la reducción en el tiempo de cursada que ofrecen las ofertas de terminalidad (reducción de 5 días de clase a 2, menos horas de cursada, modalidad a distancia) y el funcionamiento en sedes en lugar de escuelas parecieran tener un gran impacto en la mejora de los indicadores de egreso de la escuela secundaria de adultos: en el sector estatal, se pasa de 42781 alumnos en 2010 a 63827 en 2014. En apenas cinco años, la cantidad de egresados aumenta casi el $50 \%$. Sin duda, ese pico refleja la implementación de uno de esos programas: el plan Fines 2 lanzado en 2010. Sobre el deterioro educativo que implica, remitimos a otro trabajo (De Luca, 2017a). La provincia de Córdoba fue pionera en la incorporación de programas de terminalidad y de cursado a distancia. En 2010, se creó el Programa de Inclusión y Terminalidad (PIT) dirigido a jóvenes de 14 a 17 años que hubieran abandonado el secundario o que nunca hubieran iniciado sus estudios secundarios. Los jóvenes que pasan por el programa no pueden reincorporarse a la escuela común; no se trata de un programa para recuperar trayectos sino para cursarlos. A enero de 2016, el plan ya contaba con 1085 egresados y 4500 inscriptos. Un estudio sobre el programa, realizado por la Unicef y por la Universidad Nacional de Córdoba (2013), destacaba del programa constituirse en herramienta de reinserción social, pero con fallas de calidad: en la terminalidad, se aprendía menos que en la escuela común. El sistema de cursado, al igual que el Fines 2, es flexible y se permite recursar materias sin repetir ( $L a$ voz, 2014); los módulos de trabajo se dividen en $25 \mathrm{~min}$ (aduciendo déficits de atención de los alumnos cursantes). 
A juicio de los autores, el análisis realizado permite arribar a conclusiones importantes. En primer lugar, para el nivel primario, la relectura de los datos educativos que incorporan datos poblacionales y de rendimiento educacional mostró que el aumento de la privatización responde más bien a una "ficción" estadística empujada por la política educativa. En el nivel primario, la evolución de la gestión privada no se comprende sin justipreciar la caída de la sobreedad, de la repetición, del aumento de la promoción efectiva y sin depurar la mejora en el abandono interanual del sector público, que llegó a niveles récord. Los indicadores hacen que el $17 \%$ de la matrícula estatal circule en forma más rápida por el sistema, mientras el 1,5\% abandona. Así, más de medio millón de niños circulan más rápido por la primaria estatal. En apariencia, la matrícula cae, pero no es más que la expresión de las nuevas políticas de “inclusión”, "permanencia" y "egreso". En el nivel secundario, mientras la privatización del nivel llega a su techo máximo, la extensión del sistema educativo a través de la obligatoriedad incrementa la matrícula del sector estatal, que la absorbe de diversas maneras. Mientras en el nivel primario el Estado la hace circular más rápidamente, en el secundario la hace atravesar por distintas modalidades (de la escuela común a la de adultos) para decantar la titulación (egreso) en los programas de cursado acelerado o de terminalidad. En todo ese proceso, la trayectoria educativa de cientos de miles de alumnos se ve degradada, un proceso educativo extendido en el tiempo, fragmentado y en el que de un circuito al otro se recorta tiempo de estudios, contenidos y las condiciones de cursada en sedes también impactan la calidad de los recursos escolares puestos en juego. Llegado a este punto, conviene preguntarse por la naturaleza de este proceso.

\section{A MOdO DE BALANCE: EDUCACIÓN Y CRISIS ORGÁNICA}

Pública o privada, el fenómeno que caracteriza el sistema educativo argentino es la degradación, es decir, la descomposición del proceso pedagógico y, por ende, de la situación de quienes lo protagonizan: docentes y alumnos. Al calor de la profundización de la crisis social, asistimos a una ampliación de ese proceso. Por eso, resulta difícil pensar en la privatización como negocio y, más bien, emergen formas nuevas de "estatización" y fragmentación. Población que no ingresa a la escuela, o lo hace por más años, pero obtiene peores resultados, tal como muestran todas las pruebas realizadas (Aprender, One, PISA, Terce), pérdida del poder adquisitivo del salario docente (De Luca, 2016, 2017b), deterioro de la infraestructura escolar (De Luca, 2017c), son algunas de las marcas de la crisis. Este artículo abordó una de sus caras: las formas que asume la reconfiguración de la escuela, centralmente pública, en la que la privatización difícilmente pueda hacer mella. En ese sentido, fueron las políticas de descentralización escolar, esto es, la asunción por parte de las provincias de las decisiones en materia educativa, curricular, presupuestaria y salarial, introducidas en la segunda mitad del siglo XX, las que aseguraron "flexibilidad" para el sistema. En general, la preocupación por la privatización ha impedido ver cómo operan esas tendencias en el sistema. Por cuestiones de espacio, remitimos al lector a una serie de trabajos alusivos (De Luca, 2011, 2013, 2016; De Luca y Prieto, 2016). Se sostiene en este artículo que esos cambios 
educativos responden bien a las dos grandes tendencias que siguen las relaciones sociales de producción en el país: un proceso de expansión y otro de descomposición.

Argentina es un país capitalista chico, agrario, cuyo ingreso al mercado mundial se da en forma tardía. El país logró compensar el atraso relativo en el ingreso al mercado con la obtención de una renta absoluta y diferencial, de modo que es la renta agraria el mecanismo de compensación que le permitió actuar como si fuera un país con mayor productividad del trabajo y de mayor composición orgánica del capital. Durante el auge de la renta, entre 1880 y 1930, vivió su mejor momento: desarrolló una industria local nada despreciable y un amplio mercado interno. Pero los límites de su escala y su escasa productividad hicieron que se insertara allí donde las ventajas de su localización y las peculiaridades del mercado se lo permitían: en el sector agrario. Hasta la década de los cuarenta la expansión de un aparato industrial ineficiente a escala internacional no ofrecía mayores problemas (Kabat, 2005; Sartelli, 2007, 2018). ${ }^{11}$

Durante ese proceso expansivo, la escuela argentina se prefiguró con ciertas características impuestas por el "ideario sarmientino". La educación debía ser masiva y popular, común, "libre de las odiosas diferencias entre ricos y pobres" y pública, por el lugar que desempeñaría el Estado como agente educador (Sarmiento, 2011, p. 47). Desde sus orígenes, la educación fue pensada como un elemento civilizador asociado

11 Con esto no queremos decir que el capitalismo argentino no sea lo suficientemente desarrollado. La tecnificación del agro y la escala de gran industria de varias ramas dan cuenta de su carácter acabado y de la ausencia de tareas nacionales en nuestro país. al desarrollo de las fuerzas productivas y de sus necesidades. A la necesidad de homogeneizar a la población se acompañó con un sistema fuertemente centralizado y homogéneo. Mientras la sociedad avanzó, la educación acompañó ese proceso. No solo permitió dejar atrás una sociedad analfabeta, sino que habilitó ejemplos de promoción social. Según los censos nacionales, hacia 1869 la tasa de analfabetismo del país era de 77,4; hacia 1895, de 53,3; en 1914, de 35,9; y en 1947, de 13,9 (Ministerio de Educación y Justicia, 1965, pp. 7-45). Para la misma época, el promedio de América Latina se ubicaba entre el 40 y el $50 \%$. En efecto, la Argentina se distinguió por su alto porcentaje de reclutamiento escolar: hacia fines de la década de los sesenta cerca del $94 \%$ de la población en edad escolar se incorporaba efectivamente a la escuela. Para 1950, el 85 \% de la población de más de diez años sabía leer y escribir; el promedio de América Latina se ubicaba en el 58 \% y en los Estados Unidos en un $96 \%$ (Instituto de Ciencias de la Educación, 1964, p. 20). Pero también tenía límites. La deserción era alta: en el ámbito urbano, el 39 \% de la población desertaba, el $17 \%$ ya ni siquiera se matriculaba en segundo grado. La matrícula del nivel secundario era más bien acotada: si hacia 1958 se registraban 2859826 alumnos en el nivel primario, la matrícula secundaria era un $18 \%$ (505 173 alumnos). El sistema realizaba "selecciones internas", pero, a pesar de sus déficits, la escuela primaria brindaba una formación sólida.

El agotamiento de esa sociedad también resignificó el lugar de la escuela. Durante la segunda mitad del siglo XX, el crecimiento del peso del aparato industrial y la tendencia a la disminución de la renta como 
mecanismo de compensación van a constituirse en un techo del desarrollo argentino. A partir de la década de los cincuenta, la sociedad argentina empieza a expulsar población, su clase obrera se fragmenta. Los límites de un país atado a la producción agraria, rama que se retrasa permanente en el mercado mundial con una industria de escasa escala, cuya productividad se lo hace en comparación con la internacional, marcan los límites al desarrollo de las relaciones sociales capitalistas y el inicio de su descomposición. La renta que operaba como factor de compensación se achica y emergen centralmente dos que marcarán la dinámica posterior: la inflación, mecanismo que permite aumentar la tasa de explotación rebajando el salario y la deuda. Crisis recurrentes que no hacen más que agravarse con el tiempo: 1975, 1982, 1989, 2001, 2008. Todos esos episodios fueron precedidos de una caída de la producción agraria, de una reducción importante del capital industrial obsoleto y de una consolidación de masas cada vez mayores de población "sobrante". En ese proceso, la Argentina cede la primacía económica en América Latina a manos de Brasil y México.

A partir de la década de los sesenta $\mathrm{y}$, con mayor fuerza, desde la década de los setenta, al calor de la escasez de recursos, los pedagogos comenzaron a medir el rendimiento de la escuela. Se revisó lo actuado y una de las primeras propuestas fue dejar atrás la homogeneidad. Se dio paso a la descentralización y se inició la desarticulación completa del sistema. Así, se abrió paso a la descentralización. El proceso convivió con tendencias a la ampliación de la obligatoriedad escolar. Como se mostró, ese proceso no fue exitoso (ni desde el punto de vista de garantizar cobertura ni de rendimientos) y, en realidad, el sistema profundizó vías para procesar esa crisis: población que circula de las modalidades tradicionales a las de adultos aparecen como "fuga hacia adelante".

Un informe de la Unesco (2001) destacaba que, si se comparaban dos cohortes educativas, una de inicios de la década de los sesenta (1961-1962) y otra de mediados de la década de los noventa (1996-1997), los indicadores retrocedían. En términos relativos, el abandono en séptimo grado era mayor en 2001 que treinta y cinco años atrás. Algo similar ocurría en el nivel secundario. La política educativa se encargó de procesar ese problema pos-2003 ocultándolo. El impacto es mayor en el circuito estatal y por eso la tendencia pareciera ser la privatización. Pero, como se mostró aquí, el proceso parece ser el contrario: mayor estatización bajo nuevas formas que buscan dar respuesta a la crisis orgánica de la sociedad. Los pobres resultados deberían llamar la atención sobre la urgente necesidad de una vía alternativa.

\section{Referencias}

Almeida, M. y Pok C. (2014). No somos cómplices de la mentira: los trabajadores del Indec denuncian la destrucción de las estadísticas públicas tras siete años de intervención. Buenos Aires, Argentina: CTA.

Ball, S. (2014). Globalización, mercantilización y privatización: tendencias internacionales en educación y política Educativa. Education Policy Analysis Archives, 22(41), 1-13.

Ball, S. y Youdell, D. (2007). Privatización encubierta en la educación pública. Recuperado de http://www.unionferroviaria.uy/wp-content/uploads/2017/04/ 
Ball_S._y_Youdell_D._2008_La_privatizaci\%C3\%B3n_encubierta_en_la_educaci $\%$ C3\%B3n_p\%C3\%BAblica1.pdf

Braverman, H. (1975). Trabajo y capital monopolista: la degradación del trabajo en el siglo XX. Buenos Aires, Argentina: Nuestro Tiempo.

Consejo Latinoamericano de Ciencias Sociales (Ed.) (2005). Las reformas educativas en los países del Cono Sur: un balance crítico. Buenos Aires, Argentina: Autor.

De Luca, R. (2011). Onganía y la Ley Orgánica de Educación: currículum flexible, personalización de la enseñanza y atributos productivos (1966-1972). En E. Sartelli (Comp.), La crisis orgánica de la sociedad argentina (pp. 119-170). Buenos Aires, Argentina: Universidad de Buenos Aires.

De Luca, R. (2013). La construcción de una ideología educativa: diagnósticos y propuestas de reforma educativa en Argentina. Revista Actualidades Investigativas en Educación, 13(3), 1-30.

De Luca, R. (2016). El retorno del analfabetismo a la Argentina: titularización y desempeño escolar en la década kirchnerista. Razón y Revolución, 29, 199-226.

De Luca, R. (2017a). Brutos y baratos: descentralización y privatización en la educación argentina (1955-2001). Buenos Aires, Argentina: Ediciones RYR.

De Luca, R. (2017b). Una mirada histórica sobre la evolución del salario docente, desde la década del 30 hasta la actualidad. Ponencia presentada en XI Jornadas de Historia Argentina y Latinoamericana, Buenos Aires, Argentina.

De Luca, R. (2017c). Una primera aproximación al conflicto social educativo en la provincia de Salta, 2003-2015. En G. Galafassi y S. Puricelli (Comp.), Perspectivas críticas sobre la conflictividad social (pp. 133-154). Quilmes, Argentina: Theomai.

De Luca, R. y Prieto, N. A. (2016). Un análisis comparativo de las reformas educativas orgánicas de los años sesenta y noventa en Argentina: ¿transformación o consolidación de tendencias? Cadernos de História da Educação, 15(1), 378-397.

Dirección Nacional de Información y Evaluación de la Calidad Educativa. (2003). Anuario estadístico educativo. Buenos Aires, Argentina: Ministerio de Educación. Recuperado de https://www.argentina. gob.ar/educacion/planeamiento/info -estadistica/anuarios

Dirección Nacional de Información y Evaluación de la Calidad Educativa (2014). Anuario estadístico educativo. Buenos Aires, Argentina: Ministerio de Educación. Recuperado de https://www.argentina.gob.ar/educacion/planeamiento/ info-estadistica/anuarios

Dirección Nacional de Información y Evaluación de la Calidad Educativa. (2015). Pedido de Información. Ref. Nota 822/15. Mecanografiado suministrado a la autora. Buenos Aires, Argentina: Autor.

Feldfeber, M. (2006). Reforma educativa y regulación estatal: los docentes y las paradojas de la autonomía impulsada por decreto. En M. Feldfeber y D. Andrade Oliveira (Comp.), Politicas educativas y trabajo docente: nuevas regulaciones, ¿nuevos sujetos? (pp. 53-72). Buenos Aires, Argentina: Noveduc.

Feldfeber, M. (2009). Nuevas y viejas formas de regulación del sistema educativo en Argentina. Linhas Críticas, 15(28), 25-43. Recuperado de http://www.redalyc. org/pdf/1935/193514385002.pdf

Feldfeber, M. y Gluz, N. (2011). Las políticas educativas en Argentina: herencias 
de la década de los noventa, contradicciones y tendencias de "nuevo signo". Educação \& Sociedade, 32(115), 339-356. Recuperado de http://www.redalyc. org/html/873/87319092006/

Fondo de las Naciones Unidas para la Infancia. (2003). Informe anual del Unicef 2003. Recuperado de https://www.unicef.org/spanish/publications/files/2628_ spa_final.pdf

Fondo de las Naciones Unidas para la Infancia. (2005). Informe anual del Unicef 2005. Recuperado de https://www.unicef.org/spanish/publications/files/Informe_Anual_2005.pdf

Fondo de las Naciones Unidas para la Infancia. (2007). Informe anual 2007. Recuperado de https://www.unicef.org/ spanish/publications/files/Informe_ Anual_2007.pdf

Fondo de las Naciones Unidas para la Infancia. (2013). Programa de Inclusión y Terminalidad de la Educación Secundaria para Jóvenes de 14 a 17 años (PIT) Córdoba, Argentina. Autor.

Fondo de las Naciones Unidas para la Infancia. (s. f.). Informe nacional: las oportunidades educativas en la Argentina 1998-2010. Buenos Aires, Argentina: Autor. Recuperado de http://www.educacionparatodos.org.ar/oportunidades/ Documentos/Informe\%20nacional $\% 20$ -\%20Las\%20oportunidades\%20educativas\%20en \%20Argentina\%20(1998\%20 -\%202010).pdf

Filmus, D. (1997). Estado, sociedad y educación en la Argentina de fin de siglo: proceso y desafíos. Buenos Aires, Argentina: Troquel.

Fimus, D. (2000). La educación latinoamericana: entre la transformación y el ajuste. En G. Frigerio, M. Poggi y M. Giannoni
(Comps.), Políticas instituciones y actores en educación (pp. 53-64). Buenos Aires, Argentina: Novedades Educativas.

Friedman, M. (1997). Public schools: Make them private. Education Economics, 5(3), 341-344.

Gamallo, G. (2015). La "publificación" de las escuelas privadas en Argentina. Revista $S A A P, 9(1), 43-74$. Recuperado de http://www.scielo.org.ar/scielo.php?pi$\mathrm{d}=$ S1853-19702015000100002\&script=sci_arttext\&tlng=en

Ganimian, A. (2014). Avances y desafíos pendientes: informe sobre el desempeño de Argentina en el Tercer Estudio Regional Comparativo y Explicativo (TERCE) del 2013. Buenos Aires, Argentina: Organización de las Naciones Unidas para la Educación, la Ciencia y la Cultura.

Guadagni, A. (2013a, septiembre 2). Una década de retroceso de la escuela pública. Clarín. Recuperado de https://www.clarin.com/opinion/decada-retroceso-escuela-publica_0_Bk9gwo8ovXl.html

Guadagni, A. (2013b, septiembre 5). La educación argentina frente al mundo globalizado. Consejo Argentino para las Relaciones Internacionales. Recuperado de http://www.cari.org.ar/recursos/cronicas/guadagni5-9-13.html

Instituto Nacional de Estadísticas y Censos. (1994). Situación y evolución social. Síntesis (2). Buenos Aires, Argentina: Autor.

Instituto Nacional de Estadísticas y Censos. (2004). Estimaciones y proyecciones de población. Total del país: 1950-2015. Buenos Aires, Argentina: Autor.

Instituto Nacional de Estadísticas y Censos. (2001). Metodología para el ajuste de las tasas de escolarización a partir de la información del Censo Nacional de Población, 
Hogares y Viviendas de 2001. Buenos Aires, Argentina: Autor.

Infobae. (2015, octubre 3). Los engaños del Indec y las cifras del desempleo. Recuperado de https://www.infobae.com/ 2015/10/03/1759839-los-enganos-del-indec-y-las-cifras-del-desempleo/

Instituto de Ciencias de la Educación. (1964). Deserción escolar primaria en la Argentina: informe de investigación N. 1 . Buenos Aires, Argentina: Facultad de Filosofía y Letras.

Junta Interna ATE-Indec. (2010a). Censo 2010: lo que no se va a poder contar. Recuperado de http://www.ateindec.org.ar/ documentos/55\%20CENSO_2010\%20 $10 \% 20$ que $\% 20$ no $\% 20$ se $\% 20$ va $\% 20$ poder\%20contar.pdf

Junta Interna ATE-Indec. (2010b). ¡Que lindo, que lindo que va a ser, el Censo maquillado en el Sheraton Hotel! Recuperado de http://www.ateindec.org. ar/documentos/83\%20SHERATON\%20 PILAR\%20CENSOque\%20lindo...pdf

Kabat, M. (2005). Del taller a la fábrica: proceso de trabajo, industria y clase obrera en la rama del calzado (Buenos Aires 1870-1940). Buenos Aires, Argentina: Ediciones RyR. Kabat, M. (2007). Secundario completo: las demandas actuales del capital en materia educativa. En E. Sartelli (Comp.), Contra la cultura del trabajo: una crítica marxista del sentido de la vida en la sociedad capitalista. Buenos Aires, Argentina: Ediciones RyR.

La Gaceta. (2007, marzo 20). Se redujo el fracaso escolar en cinco provincias. Recuperado de http://www.lagaceta.com. ar/nota/198733/se-redujo-fracaso-escolar-cinco-provincias.html

La Nación. (2014, octubre 2014). El ministro Alberto Sileoni aseguró que el éxodo hacia escuelas privadas se debe al "crecimiento económico". Recuperado de https://www.lanacion.com.ar/ 1739991-el-ministro-alberto-sileoni-aseguro-que-el-exodo-hacia-escuelas-privadas-se-debe-al-crecimiento-econo mico

La Voz. (2011, septiembre 1). Argentina tiene 40.117.096 habitantes, según datos definitivos del Censo 2010. Recuperado de http://www.lavoz.com.ar/ciudadanos/argentina-tiene-40117096-habitantes-segun-datos-definitivos-censo-2010

La Voz. (2014, abril 15). Vuelven a la escuela y se integran socialmente, pero aprenden poco. Recuperado de http:// www.lavoz.com.ar/ciudadanos/vuelven-la-escuela-y-se-integran-socialmente-pero-aprenden-poco

Marrone, L. (2014). A más de dos décadas de reformas: la educación, más privada y menos pública. Ponencia presentada en III Congreso Nacional de Educación, Buenos Aires, Argentina.

Ministerio de Educación. (2012). Una escuela más justa produce mejores resultados. Repitencia y promoción en primer grado: las dos caras de una moneda. Buenos Aires, Argentina: Autor.

Ministerio de Cultura y Educación. (1973). La educación en cifras. 1963-1972. (t. I y II). Buenos Aires, Argentina: Autor.

Ministerio de Cultura y Educación. (1974). Establecimientos, alumnos y docentes por dependencia, repartición y jurisdicción. 1964-1973, Buenos Aires, Argentina. Autor.

Ministerio de Cultura y Educación (1975). Estadísticas de la educación. Síntesis. 19731977. Buenos Aires, Argentina: Autor.

Ministerio de Cultura y Educación (1978). Estadísticas de la educación. Establecimientos, 
alumnos, docentes: cifras provisorias. 1978, Buenos Aires, Argentina: Autor.

Ministerio de Cultura y Educación (1979). Estadísticas de la educación. Establecimientos, alumnos, docentes: cifras estimadas. Buenos Aires, Argentina: Autor.

Ministerio de Cultura y Educación (1980). Establecimientos, alumnos, docentes: cifras provisorias. Buenos Aires, Argentina: Autor.

Ministerio de Cultura y Educación (1981). Estadísticas de la educación. Establecimientos, alumnos, docentes: cifras estimadas. Buenos Aires, Argentina: Autor.

Ministerio de Cultura y Educación. (1982). Estadísticas de la educación. Establecimientos, alumnos, docentes: cifras estimadas. Buenos Aires, Argentina: Autor.

Ministerio de Cultura y Educación. (1983). Estadísticas de la educación. Establecimientos, alumnos, docentes: cifras provisionales. Buenos Aires, Argentina: Autor.

Ministerio de Cultura y Educación. (1984). Estadísticas de la educación. Establecimientos, alumnos, docentes: cifras provisionales. Buenos Aires, Argentina: Autor.

Ministerio de Cultura y Educación (1985). Estadísticas de la educación. Establecimientos, alumnos, docentes. Cifras provisionales. Buenos Aires, Argentina: Autor.

Ministerio de Cultura y Educación (1986). Estadísticas de la educación. Establecimientos, alumnos, docentes: cifras provisionales. Buenos Aires, Argentina: Autor.

Ministerio de Cultura y Educación (1987). Estadísticas de la educación. Establecimientos, alumnos, docentes: cifras provisionales. Buenos Aires, Argentina: Autor.

Ministerio de Cultura y Educación (1996). Anuario estadístico educativo. Buenos Aires, Argentina: Autor.
Ministerio de Cultura y Educación (1997). Anuario estadístico educativo. Buenos Aires, Argentina: Autor.

Ministerio de Cultura y Educación (1999). Anuario estadístico educativo Buenos Aires, Argentina. Autor.

Ministerio de Cultura y Educación. (2000). Anuario estadístico educativo. Buenos Aires, Argentina: Autor.

Ministerio de Cultura y Educación. (2002). Anuario estadístico educativo. Buenos Aires, Argentina: Autor.

Ministerio de Cultura y Educación. (2003). Anuario estadístico educativo. Buenos Aires, Argentina: Autor.

Ministerio de Educación, Cultura, Ciencia y Tecnología (2002-2013). Anuarios estadísticos. Recuperado de https:// www.argentina.gob.ar/educacion/ planeamiento/info-estadistica/anuarios

Ministerio de Educación y Deportes. (2012). El nivel primario: un análisis cuantitativo. Buenos Aires, Argentina: Autor. Recuperado de http://repositorio.educacion. gov.ar:8080/dspace/bitstream/handle/123456789/109828/Serie\%20investigaci\%C3\%B3n\%20web\%207.pdf

Ministerio de Educación de la Nación (2013). La educación argentina en cifras 2012: 15 años del relevamiento anual. Buenos Aires, Argentina: Autor.

Ministerio de Educación y Justicia. (1965). Informe de la República Argentina. En Congreso Mundial de Ministros de Educación para la Liquidación del Analfabetismo, Teherán, Irán.

Morduchowicz, A., Marcon, A., Iglesias, G., Andrada, M., Pérez, J., Campan, V. y Duro, L. (1999). La educación privada en la Argentina: historia, regulaciones y asignaciones de recursos públicos. Buenos Aires: Ministerio de Educación. 
Narodowski, M. (2008). School choice and quasi-state monopoly in education systems in Latin America: The case of Argentina. En M. Forsey, S. Davies \& G. Walford (Eds.), The globalisation of school choice? (pp. 131-144). Oxford, RU: Symposium Books.

Naradowski, M. y Andrada, M. (2001). The privatization of education in Argentina. Journal of Education Policy, 16(6), 585-595.

Narodowski, M. y Moschetti, M. (2014, julio 15). ¡Vuelen, blancas palomitas! La caída de la matrícula en las escuelas primarias públicas argentinas [Entrada blog]. Recuperado de http://focoeconomico. org/2014/07/15/vuelen-blancas-palomitas-la-caida-de-la-matricula-en-las-escuelas-primarias-publicas-argentinas/

Narodowski, M. y Moschetti, M. (2015a). The growth of private education in Argentina: Evidence and explanations. Compare: A Journal of Comparative and International Education, 45(1), 47-69.

Narodowski, M. y Moschetti, M. (2015b). Why does private school enrollment grow? Evidence from Argentina. Cogent Education, 2(1). Recuperado https:// www.tandfonline.com/doi/abs/10.1080/ 2331186X.2015.1077604

Narodowski, M. y Nores, M. (2000). ¿Quiénes quedan y quiénes salen? Características socioeconómicas en la composición de la matrícula en las escuelas públicas y en las escuelas privadas en la Argentina. Buenos Aires, Argentina: Fundación Gobierno y Sociedad.

Narodowski, M., Moschetti, M. Alegre, S. (2016). Teacher strikes and private education in Argentina. Revista Colombiana de Educación, 70, 27-45. Recuperado de http://www.scielo.org.co/scielo.
php?pid=S0120-39162016000100003\&script=sci_abstract\&tlng=pt

Narodowski, M., Moschetti, M. y Gottau, V. (2017). El crecimiento de la educación privada en Argentina: ocho explicaciones paradigmáticas. Cadernos de Pesquisa, 47(164), 414-441. Recuperado de http://www.scielo.br/scielo.php?pi$\mathrm{d}=$ S0100-15742017000200002\&script=sci_abstract\&tlng=pt

Organización de las Naciones Unidas para la Educación, la Ciencia y la Cultura. (2001). La reforma educativa en la Argentina:. semejanzas y particularidades. Buenos Aires, Argentina: Autor.

Organización para la Cooperación y el Desarrollo Económico. (2003). Aptitudes básicas para el mundo de mañana: otros resultados del proyecto PISA 2000. París, Francia: Autor.

Paviglianiti, N. (1995). La Ley Federal de Educación como elemento de regulación de la realidad socioeducacional en la Argentina: Sus orientaciones hacia la privatización, provincialización y retiro del Gobierno Nacional del financiamiento del sistema de educación pública. Serie Pedagógica, 2, 123-146. Recuperado de http://www.memoria. fahce.unlp.edu.ar/art_revistas/pr.2535/ pr.2535.pdf

Perfil. (2014, octubre 5). Trabajo y pobreza. Recuperado de https://www.perfil. com/noticias/economia/Trabajo--y-pobreza-20141005-0005.phtml

Proyecto de Resolución La Honorable Cámara de Diputados. Recuperado de http://www.ferrarigustavo.com/archivos/InformeCenso.pdf

Puiggrós, A. (Dir.) (1997). Historia de la educación en la Argentina. Vol. VIII: Dictaduras y utopías en la historia reciente de 
la educación (1955-1983). Buenos Aires, Argentina: Galerna.

Puiggrós, A. (2003). Qué pasó en la educación argentina: breve historia desde la Conquista hasta el presente. Buenos Aires, Argentina: Galerna.

Resolución № 216/04 C.F.C. Y E.

Resolución CFE № 174/12.

Rivas, A. (2015). América Latina después de PISA: lecciones aprendidas de la educación en siete países (2000-2015). Buenos Aires, Argentina: CIPPEC.

Sarmiento, D. F. (2011). Educación popular. Buenos Aires, Argentina: UNIPE.

Sartelli, E. (2007). La plaza es nuestra: el Argentinazo a la luz de la lucha de la clase obrera en la Argentina del siglo XX. Buenos Aires, Argentina: Ediciones RyR.

Sartelli, E. (Comp.) (2011). La crisis orgánica de la sociedad argentina. Buenos Aires, Argentina: Universidad de Buenos Aires.

Sartelli, E. (2013). La cajita infeliz: un viaje marxista a través del capitalismo (4..$^{\mathrm{a}} \mathrm{ed}$.). Buenos Aires, Argentina: Ediciones RyR.

Sartelli, E. (2014). Ríos de oro y gigantes de acero: tecnología y clases sociales en la región pampeana (1870-1940). Razón y Revolución, 3. Recuperado de http:// www.revistaryr.org.ar/index.php/ryr/ article/view/458

Sartelli, E. (2018). La sal de la tierra. Buenos Aires, Argentina: Ediciones RyR.

Sartelli, E. y Kabat, M. (2014). Where did Braverman go wrong? A Marxist response to the politicist critiques. Cadernos EBAPE. BR, 12(4), 829-850.

Sartelli, E. y Kabat, M. (Coords.) (2017). Mentiras verdaderas: ideología, nacionalismo y represión en la Argentina 1916-2015. Buenos Aires, Argentina: Universidad de Buenos Aires.
Tenti Fanfani, E. y Grimson, A. (2014). Mitomanías de la educación argentina: crítica de las frases hechas, las medias verdades y las soluciones mágicas. Buenos Aires, Argentina: Siglo XXI.

Tiramonti, G. (1997). Descentralización y reestructuración social: ¿movimientos complementarios? En M. Noriega (Coord.), Políticas educativas nacionales y regionales. Investigación Educativa 19931995 (t. 3, pp. 24-33). Ciudad de México, México: Consejo Mexicano de Investigación Educativa/Universidad Pedagógica Nacional.

Torres, C. A. (2008). Después de la tormenta neoliberal: la política educativa latinoamericana entre la crítica y la utopía. Revista Iberoamericana de Educación, 48, 207-229. Recuperado de https://rieoei. org/RIE/article/view/697

Tribuna Docente. (2005). Las reformas capitalistas de la educación en la Argentina y el mundo. Buenos Aires, Argentina: Autor.

Vior, S. y Rodríguez, L. (2012). La privatización de la educación argentina: un largo proceso de expansión y naturalización. Pro-Posições, 23(2), 91-104. Recuperado de http://www.scielo.br/pdf/pp/v23n2/ a07v23n2

Whitty, G. y Power, S. (2000). Marketization and privatization in mass education systems. International Journal of Educational Development, 20(2), 93-107. Doi: https:// doi.org/10.1016/S0738-0593(99)00061-9 